\title{
Associação de inseticidas químicos e fungos entomopatogênicos no Manejo Integrado de Pragas - uma revisão
}

\author{
Association of chemical insecticides and entomopathogenic fungi in \\ Integrated Pest Management - a review
}

\author{
Larissa Rolim Borges ${ }^{1(*)}$ \\ Meiriana Xavier Vila Nova ${ }^{2}$
}

\section{Resumo}

O desenvolvimento rápido da resistência a inseticidas tem demonstrado a limitada eficiência desse método para a supressão de populações de insetos, e incentivado pesquisas com métodos alternativos de controle. A utilização de inseticidas em associação com entomopatógenos no manejo integrado de pragas é uma estratégia que vem se mostrando bastante eficaz para a redução da densidade populacional da praga desde que ambos sejam compatíveis. $\mathrm{O}$ uso da associação pode aumentar a eficiência do controle de insetos, permitindo a redução na quantidade de inseticidas aplicados, preservação de inimigos naturais e organismos não alvos e aumento da biodiversidade em culturas agrícolas. Porém, o sucesso desta técnica depende do uso da formulação e do propágulo correto; da presença de hospedeiros suscetíveis, condições ambientais favoráveis e cronograma compatível com outras práticas agrícolas.

Palavras-chave: agrotóxicos; fungo; controle biológico.

\section{Abstract}

The quickly development of insecticide resistance has demonstrate the limited efficiency of this method for suppression of insect population and stimulated researches with alternative methods of control. The application of insecticides in association with entomopathogens in Integrated Pest Management is a recommended strategy to reduce the population density of this pest if both products are compatible. The use of this association can increase the control efficiency,

I Dra.; Bióloga; Pesquisadora na Empresa Pernambucana de Pesquisa Agropecuária, IPA, Recife; Endereço: Rua Rubens de Oliveira Santos, 4I, Bonsucesso, CEP: 44.380-000, Cruz das Almas, Bahia, Brasil; E-mail: larissarolimborges@gmail.com (*) Autora para correspondência.

2 Dra.; Bióloga; Departamento de Micologia, Universidade Federal de Pernambuco - UFPE, Recife, PE, Brasil. E-mail: meiriana_vilanova@yahoo.com.br

Recebido para publicação em 05/02/2010 e aceito em 28/03/20 I I

Ambiência Guarapuava (PR) v.7 n.I p.I79 - $190 \quad$ Jan./Abr. 20II ISSN I808 - 025।

DOI:I0.5777/ambiencia.201 I.01.02rb 
allowing the reduction of insecticide applied, preserving the natural enemies and non-target organisms and increasing the biodiversity in "agricultural cultures". However, the success depends on the use of the correct propagule and formulation, presence of susceptible host, favorable environmental conditions and compatible schedule with other agricultural practices.

Key words: insecticides; fungi; biological control.

\section{Introdução}

A necessidade cada vez maior de produzir alimentos de alta qualidade tem levado o homem a provocar grandes alterações nos ecossistemas naturais para o plantio de monoculturas. Como principal consequência deste desequilíbrio ecológico observa-se o alto crescimento populacional de insetos que provocam danos às plantas comerciais, acarretando grandes prejuízos econômicos (ORIOLI; CAMARGO, 2000).

Ao longo de várias décadas, o uso indiscriminado de produtos fitossanitários para o controle de insetos provocou a redução da produtividade, contaminação dos alimentos e efeitos indesejáveis sobre o ambiente, sobre o homem e outros animais, e relatos de resistência a moléculas inseticidas em diversas espécies de insetos (CAVALCANTI et al., 2002; GALLO et al., 2002).

O princípio básico do Manejo Integrado de Pragas (MIP) preconiza a combinação de diferentes técnicas e recursos disponíveis para manter as populações de insetos abaixo do nível de dano econômico em culturas agrícolas e a maximização da ação dos inimigos naturais (PEDROSA-MACEDO, 1993). De acordo com Georghiou (1986) e Omoto (2000), uma estratégia promissora no MIP é a utilização de dois ou mais produtos em rotação ou mistura, conhecido como ataque múltiplo, pois a frequência de resistência dos insetos a uma classe inseticida diminui quando outro agente de controle é utilizado simultaneamente.

Assim, este trabalho tem por objetivo apresentar uma revisão bibliográfica da utilização dos fungos entomopatogênicos (controle biológico) associado a inseticidas (controle químico) visando o Manejo Integrado de Pragas.

\section{Controle Biológico com Fungos Entomopatogênicos}

O controle biológico consiste na utilização racional de patógenos (bactérias, fungos e vírus) visando à manutenção das populações de insetos não alvos (parasitóides, predadores e polinizadores). Este método pode ser integrado ao controle químico, físico (drenagem e irrigação), mecânico, por comportamento (uso de feromônios), de resistência de plantas (transgênicos) e outras estratégias (GALLO et al., 2002; PEDROSA-MACEDO, 1993).

Os fungos são definidos como organismos eucarióticos, cujos núcleos são dispersos em um micélio - conjunto de hifas - contínuas ou septadas. Não possuem pigmentos fotossintéticos e sua nutrição é obtida por absorção. Os fungos são bastante conhecidos pela produção de compostos químicos, alguns bastante potentes, causando reações adversas em outros organismos vivos, como os antibióticos e as micotoxinas (GRANDI et al., 1996). 
Fungos entomopatogênicos são aqueles que causam danos aos insetos. Aproximadamente $80 \%$ das doenças destes têm como agentes etiológicos os fungos, pertencentes a cerca de 90 gêneros e mais de 700 espécies (ALVES et al., 1998). No Brasil, mais de 20 gêneros de fungos ocorrem naturalmente sobre pragas de importância econômica, sendo os mais importantes pertencentes à classe Deuteromycetes, como Metarbizium, Beauveria, Lecanicillium, Nomurea, Aschersonia, Hirsutella, Paecilomyces e Entomophora (ALVES, 1986; SHAH e PELL, 2003).

Principalmente Beauveria bassiana (Bals.). Vuill. e Metarbizium anisopliae (Metschnikoff) Sorokin. demonstram grande potencial como agentes de controle microbiano, sendo comumente encontrados infectando mais de 200 espécies de insetos de importância agrícola, como cupins (ALMEIDA et al., 1998; MOINO JR. e ALVES, 1998), coleópteros (FURLONG; GRODEN, 2003), hemípteros (SOSAGÓMEZ et al., 1997), blatódeos (PACHAMUCHU; KAMBLE, 2000), ortópteros e pragas de grãos armazenados.

Entretanto, para que a ação desses fungos seja otimizada, são necessárias algumas condições favoráveis, destacando-se a temperatura, umidade relativa e radiação solar. A temperatura é muito importante para os fungos entomopatogênicos porque afeta seu metabolismo, alterando processos de produção de enzimas, toxinas, germinação dos esporos, desenvolvimento do tubo germinativo, penetração, colonização e reprodução. De modo geral, a faixa ideal para estes organismos varia entre 22 e 30 o C (ALVES et al., 1998).

Umidades relativas altas (entre $70 \mathrm{e}$ 100 \%) estão normalmente correlacionadas com as epizootias, pois esta é essencial para as fases de disseminação, germinação, penetração e limitante para a produção de alguns entomopatógenos. Os insetos também têm sua atividade e desenvolvimento limitado em ambiente com baixa UR \%, pois esta influi na espessura da sua camada de cera, tornando-os menos resistentes aos patógenos (ALVES et al., 1998).

A radiação ultravioleta representa o principal agente de inibição dos entomopatógenos e estes, de maneira geral, perdem sua infectividade quando submetidos a algumas horas de luz solar direta (ALVES et al., 1998).

Para Gallo et al. (2002), apesar do avanço incontestável do controle microbiano nos últimos anos, é importante mencionar que os microorganismos entomopatogênicos raramente devem ser considerados isoladamente no controle de pragas. Este tipo de controle deve fazer parte de um conjunto de medidas, as quais, atuando em harmonia com o ambiente, sejam capazes de reduzir a população de pragas e estabelecer, enzoóticamente, o patógeno no agroecossistema.

O modo de ação dos fungos entomopatogênicos é bastante versátil, causando danos a artrópodes que vivem em plantas, solos e ambientes aquáticos. Com um mecanismo de infecção especializado, alguns fungos podem infectar diferentes estágios de desenvolvimento dos hospedeiros, inclusive os estágios em que estes não se alimentam, como ovos e pupas (ALVES et al., 1998). Além disto, diferente de outros entomopatógenos, estes fungos infectam os insetos não apenas pela ingestão, mas principalmente pela penetração via tegumento e espiráculos. Esta propriedade os coloca em vantagem em relação às 
bactérias e vírus, que penetram no inseto apenas por via oral, pois a infecção pode ocorrer independente da sua alimentação (FERRON, 1978).

Em muitos casos, a infecção fúngica progride e o inseto infectado morre devido à produção de toxinas, mudanças na hemocele, ação histolítica, bloqueio mecânico do aparelho digestivo (em função do crescimento vegetativo) e outros danos físicos em decorrência do crescimento do micélio. O ciclo da relação fungohospedeiro está completo quando ocorre a esporulação no cadáver do inseto infectado, permitindo a transmissão e disseminação horizontal do entomopatógeno na população (CHARNLEY, 1997).

\section{Inseticidas químicos}

São substâncias que, aplicadas direta ou indiretamente sobre o inseto, em doses adequadas, provocam sua morte. Baseado nos processos fisiológicos ou bioquímicos envolvidos no seu modo de ação, estes podem ser divididos em neurotóxicos e reguladores de crescimento (GALLO et al., 2002).

Os inseticidas neurotóxicos têm como principal alvo de ação o sistema nervoso e apresentam alta eficácia e rapidez para o controle de pragas. O primeiro grupo de inseticidas desta classe, amplamente utilizados na agricultura, foram os organoclorados (DDT, BHC, ciclodienos, metoxicloro e policloroterfenos, entre outros) no início de 1940, época da Segunda Guerra Mundial (WARE, 2010). Entretanto, a conscientização dos problemas acarretados pelo uso indiscriminado destes agrotóxicos, assim como sua elevada toxicidade, retenção nos tecidos adiposos animais e a longa permanência no ambiente, levaram à sua proibição na década de 1980, no Brasil (BAPTISTA, 1990; GALLO et al., 2002).

Os organofosforados também foram descobertos na década de 1940. Estes inseticidas neurotóxicos atuam na transmissão sináptica levando à hiperexcitação do sistema nervoso. Em geral são muito tóxicos em exposições agudas, porém, amplamente utilizados na agricultura, veterinária e em domicílios (WARE, 2010). Do ponto de vista da segurança ambiental, não são significativamente tóxicos, pois apresentam curto período residual. As moléculas mais conhecidas desta classe de inseticidas são o acefato, metamidofós, monocrotofós, paration metil, triclorfon e diclorvós (ANDREI, 1999; GALLO et al., 2002).

$\mathrm{Na}$ década de 1950 foram introduzidos no mercado os inseticidas carbamatos, inibidores de acetilcolinesterase, em geral bastante tóxicos. Principalmente de uso agrícola e residencial, inclui representantes importantes como: aldicarb, carbofuran, carbosulfan, carbaril e propoxur (CASSIDA e QUISTAD, 1998; FERREIRA, 1999).

Os piretróides que surgiram na década de 1960, atuam na transmissão axônica e são bastante conhecidos pelo seu efeito de choque ("know-down") quase instantâneo nos insetos (GALLO et al., 2002). No ambiente, possuem pouca persistência (biodegradáveis). Geralmente pouco ou moderadamente tóxicos aos vertebrados, entretanto, frequentemente produzem reações alérgicas (FERREIRA, 1999; GALO et al., 2002). Exemplos importantes desta classe são alfacipermetrina, deltametrina, permetrina, cipermetrina e lambda-cialotrina (FERREIRA, 1999; MIDIO e SILVA, 1995).

Outras importantes classes de inseticidas neurotóxicos são os neonicotinóides 
- antagonistas da acetilcolina (imidacloprid, thiamethoxan, acetamiprid e spinosinas); avermectinas - agonistas do GABA (abamectina e milbemicina), ciclodienos - antagonistas do GABA (ciclodienos como endosulfan e fenil-pirazóis como fipronil) e as oxadiazinas - bloqueadores de canais de sódio (GALLO et al., 2002; TOMIZAWA e CASIDA, 2003).

Desde a década de 1960, com a seleção da resistência de pragas a inseticidas e a preocupação com seus efeitos adversos, as pesquisas foram direcionadas para a busca de novas moléculas, com alta seletividade a organismos não alvos (inimigos naturais de pragas e polinizadores) e menor persistência no meio ambiente, surgindo então os reguladores de crescimento (GALLO et al., 2002).

Os inseticidas do grupo dos reguladores de crescimento exercem sua ação tóxica em formas imaturas (larvas e ninfas), principalmente durante a ecdise. Assim, imaturos tratados com estes inseticidas não conseguem formar uma nova cutícula. Os principais representantes são as benzoilfeniluréias - (diflubenzuron, flufenoxuron, lufenuron, nuvaluron, teflubenzuron e triflumuron) (CASSIDA e QUISTAD, 1998; GALLO et al., 2002; REYNOLDS, 1987). De acordo com Romero apud Faria (2009), existem grandes benefícios nesta classe de inseticidas, como sua alta eficiência, baixa toxicidade para vertebrados e seletividade para o meio ambiente.

Apesar das características menos agressivas de algumas classes de inseticidas, o uso indiscriminado destes tem acarretado diversos problemas ao longo do tempo (GALLO et al., 2002). Em função disto, atualmente são comuns relatos da resistência de insetos em praticamente todos os grupos de agroquímicos, inclusive os reguladores de crescimento e produtos de origem microbiana, como o Bacillus thuringiensis e Baculovirus anticarsia (OMOTO e ALVES, 1998; OMOTO, 2000).

Dentre as principais consequências da seleção da resistência estão, além da aplicação mais frequente dos agrotóxicos, o aumento na dosagem do produto, uso de misturas indevidas ou sua substituição por outro, geralmente de maior toxicidade (GEORGHIOU, 1983). Esses fatores comprometem os programas de MIP em vista da maior contaminação do ambiente, destruição de organismos benéficos e aumento nos custos de produção (GALLO et al., 2002).

\section{Associação de Fungos e Químicos}

Nessa associação, espera-se que o agroquímico atue como agente estressor na população praga para aumentar a eficácia do micopatógeno, contribuindo para o controle do inseto praga (ALVES et al., 1998; FERRON, 1978).

As pesquisas visando detectar os efeitos dos agrotóxicos sobre entomopatógenos iniciaram após a Segunda Guerra Mundial, com a utilização de produtos organoclorados em larga escala. Hall e Dunn (1959) mencionaram que várias espécies de fungos entomopatogênicos apresentaram diferentes reações a produtos químicos. Em 1982, o Dr. Walter M. Zeck, membro do grupo de pesquisas da Bayer, Florida, USA, observou que doses subletais de inseticidas neonicotinóides aumentavam a suscetibilidade de cupins subterrâneos a diversos fungos oportunistas, incluindo $B$. bassiana, $M$. anisopliae, Paecilomyces farinosus (Hom ex SF Gray) Brown \& Smith, Actimomucor sp. entre outros. 
Após esta descoberta, vários estudos começaram a ser realizados. Anderson e Roberts (1983) misturaram B. bassiana a fenvalerate por várias horas, constando a inibição da germinação do entomopatógeno, sugerindo que a exposição prolongada do entomopatógeno ao químico pode prejudicar o desempenho desta técnica.

Em Diaprepes abbreviatus (L.) (Coleoptera: Curculionidae), os conídios de $B$. bassiana, que atacam a cutícula, são normalmente removidos durante a ecdise. Entretanto, subdoses do inseticida imidacloprid reduziram o número de ecdises larvais e o tempo letal. Observou-se, portanto, que o sinergismo do neonicotinóide com o fungo, para o controle do inseto, é ocasionado, em parte, pela modificação do comportamento larval, envolvendo uma redução ou perda temporária da mobilidade em função da neurotoxicidade do inseticida (BATISTA FILHO et al., 2001; ELBERT et al., 1991; QUINTELA e MCCOY, 1997,1998a,b).

Neves e Alves $(1999,2000)$ em estudo realizado com Cornitermes cumulans (Kollar) (Isoptera: Termitidae) comprovaram a inibição do comportamento de limpeza deste quando tratado com conídios dos fungos entomopatogênicos $B$. bassiana e M. Anisopliae, seguido de subdosagens de imidacloprid. Este inseticida afeta a atividade normal do inseto e permite a aderência e germinação do fungo entomopatogênico na cutícula do inseto, aumentado a eficiência de infecção do patógeno.

Em estudo de Alves et al. (2001), os compostos thiacloprid (neonicotinóide) e imidacloprid em subdosagens foram compatíveis com $B$. bassiana para o controle de Bemisia tabaci (Genn.) (Homoptera: Aleyrodidae). A redução da concentração destes inseticidas em 50 a $70 \%$ representammaior economia e menor seleção da resistência na população desta praga.

Furlong e Groden (2001) detectaram sinergismo quando larvas de Leptinotarsa decemlineata (Say) (Coleoptera: Chrysomelidae) foram contaminadas com B. bassiana e, posteriormente, com subdoses de imidacloprid. Este estudo ressalta a importância da aplicação do inseticida químico anteriormente ao entomopatógeno e constata a vulnerabilidade do inseto ao ataque fúngico quando este é submetido, anteriormente, a um agente químico estressor.

Em trabalho realizado por Marquini et al. (2003), verificou-se que imidacloprid foi bastante seletivo a duas espécies de trips, Thrips tabaci Lindeman e Caliothrips brasiliensis (Morgan) (Thysanoptera: Thiripidae) e à cigarrinha Empoasca kraemeri (Ross \& Moore) (Homoptera: Cicadellidae), não afetando predadores e parasitóides comumente associados às pragas da cultura.

Vásquez et al. (2004) comprovaram, em laboratório, a compatibilidade de B. bassiana com triflumuron (regulador de crescimento), com relação aos aspectos de germinação dos conídios e crescimento micelial nas diversas concentrações testadas, apresentando-se como alternativa promissora para o controle de insetos pragas de importância agrícola e médica.

Entretanto, Benz (1971), citado por Quintela (1996), sugere que o sinergismo entre inseticida químico e biológico é apenas temporal, isto é, a combinação de ambos apenas reduz o tempo de mortalidade se comparado com os tratamentos sozinhos.

Alguns autores ressaltam que a utilização de agroquímicos incompatíveis afeta negativamente o crescimento e esporulação dos entomopatógenos (CAVALCANTI 
et al., 2002; IGNOFFO et al., 1975; GARDNER e STOREY, 1985; MALO, 1993; TAMAI et al., 2002). Entretanto, a maioria destes estudos é conduzido "in vitro”, expondo ao máximo o microrganismo à ação do químico. Segundo Alves et al. (1998), este fato não ocorre a campo, onde fatores bióticos e abióticos protegem o fungo e aumentam sua eficácia sendo, portanto, necessária a realização de novos estudos de compatibilidade a campo.

O uso do controle microbiano de insetos e sua associação ao químico, segundo Alves et al. (1998), apresentam diversas vantagens. Entre elas, a capacidade de multiplicação e dispersão do patógeno no ambiente (através dos insetos praga e de seus inimigos naturais), permitindo a sua permanência por um longo período. Além disto, após o seu estabelecimento na cultura, a doença fúngica assume caráter enzoótico nas populações de inseto. Nesta fase, o inseto dificilmente ocasiona nível de dano econômico.

Esta associação apresenta-se como estratégia eficiente para o manejo da resistência por ataque múltiplo, pois os insetos dificilmente se tornam resistentes aos patógenos que ocorrem naturalmente no meio (OMOTO, 2000). Em campo, a diferença de comportamento do inseto, pela ação do químico, é rapidamente percebida pelo agricultor, estimulando a implantação efetiva de um programa de manejo integrado.

Além da mortalidade direta, secundariamente, estudos verificaram grande interferência na capacidade reprodutiva das fêmeas, como a diminuição da oviposição e viabilidade dos ovos, e aumento da sensibilidade da população da praga a outros agentes biológicos e químicos (MULOCK; CHANDLER, 2001).
Alguns fungos são bastante específicos e altamente patogênicos para determinadas espécies e desse modo, fazem a manutenção das populações de inimigos naturais, como parasitos, predadores e polinizadores, imprescindíveis para a manutenção e preservação ambiental (ALVES et al., 1998).

$\mathrm{O}$ uso de produtos biológicos pode ser limitado por condições abióticas. Entretanto, pesquisas com formulação fúngica, ou seja, mistura do fungo a outros ingredientes têm favorecido cada vez mais a sua viabilidade, eficácia e reduzido sua sensibilidade à radiação ultravioleta, entre outros (PRIOR et al., 1988).

\section{Considerações Finais}

As perspectivas para a adoção da associação de subdosagens de agroquímicos e entomopatógenos são bastante promissoras, visto que, estudos realizados não apontam sérias contaminações aos vertebrados ou dos recursos naturais, como água e solo, sendo, porém, imprescindível a realização de monitoramento ambiental.

Diversos autores citam que é pequena a ocorrência de resíduos de pesticidas químicos nos alimentos quando estes são empregados em subdosagem, entretanto, é fundamental a obediência ao período de carência, intervalo de segurança e registro do produto à cultura. Além disso, cuidados na aplicação e correta utilização de equipamentos de segurança devem ser aliados ao treinamento e conscientização dos produtores rurais.

É importante, a realização permanente de testes com microorganismos sob a influência de produtos químicos, visando à detecção de alterações fisiológicas que acarretem a perda de patogenicidade e/ ou virulência do fungo após a aplicação do defensivo químico. 


\section{Referências}

ALMEIDA, J. E. M.; ALVES, S. B.; MOINO JR. A. E.; LOPES, R. B. Controle do cupim subterrâneo Heterotermes tenuis (Hagen) com iscas Termitrap impregnadas com inseticidas e associadas ao fungo entomopatogênico Beauveria bassiana (Bals.) Vuill. Anais da Sociedade Entomológica do Brasil. Londrina, v.27, n.4, p. 639-644, 1998.

ALVES, S. B. Fungos no controle biológico de pragas. In: ENCONTRO SUL-BRASILEIRO DE CONTROLE BIOLÓGICO DE PRAGAS, 1., 1986, Passo Fundo. Anais... Passo Fundo: AEAPF/EMBRAPA-CNPT, 1986. p. 179-189.

ALVES, S. B.; MOINO JR., A.; ALMEIDA, J. E. M. Produtos fitossanitários e entomopatógenos. In: ALVES, S. B. (Org.). Controle microbiano de insetos. 2 ed. Piracicaba: FEALQ, 1998. p. 217-238.

ALVES, S. B.; SILVEIRA, C. A.; LOPES, R. B.; TAMAI, M.A.; RAMOS, E. Q.; SALVO, S. Eficácia de Beauveria bassiana, imidacloprid e thiacloprid no controle de Bemisia tabaci e na incidência do BGMV. Manejo Integrado de Plagas, Costa Rica, n. 61, p. 31-36, 2001.

ANDERSON, T. E.; ROBERTS, D. W. Compatibility of Beauveria bassiana isolates with insecticide formulations used in Colorado potato beetle (Coleoptera: Chrysomelidae) control. Journal of Economic Entomology, v.76, n. 6, p. 1437-1441, 1983.

ANDREI, E. Compêndio de defensivos agrícolas. 6 ed. SP. In: ANDREI, E. (Org.). 1999. $672 \mathrm{p}$.

BAPTISTA, G. C. Seletividade de inseticidas e manejo integrado de pragas. In: CROCOMO, W. B. (Org.) Manejo Integrado de pragas. São Paulo: Unesp, 1990. p. 1999-2013.

BATISTA FILHO, A.; ALMEIDA, J. E. M.; LAMAS, C. Effect of Thiamethoxam on entomopathogenic microorganisms. Neotropical Entomology, Londrina, v. 30, n. 3, p. 437-447, 2001.

CASIDA, J. E.; QUISTAD, G. B. Golden age of inseticide research: past, present or future? Annual Review Entomology, v. 43, p. 1-16, 1998.

CAVALCANTI, R. S.; MOINO JR., A.; SOUZA, G. C.; ARNOSTI, A. Efeito dos produtos fitossanitários Fenpropatrina, Imidaclopride, Iprodione e Tiametoxam sobre o desenvolvimento do fungo Beauveria bassiana (Bals.) Vuill.Arquivos do Instituto Biológico, São Paulo, v. 69, n. 3, p. 17-22, 2002.

CHARNLEY, A. K. Entomopathogenic fungi and their role in peste control. Chapter 12. In: The Mycota IV - Environmental and Microbial Relationschips. Wicklow/Söderström: Eds. Springer-Verlag Berlin Heidelberg. p. 186-198, 1997. 
ELBERT, A.; BECKER, J. B.; HARTWIG, J.; ERDELEN, C. Imidacloprid - a new systematic insecticide. Pflanzenshutz-Nachr. Bayer, v. 44, p. 113-136, 1991.

FARIA, A. B. C. Revisão sobre alguns grupos de inseticidas utilizados no manejo integrado de pragas florestais. Ambiência, Guarapuava, v. 5, n. 2, p. 345-358, 2009.

FERREIRA, W. L. B. Inseticidas de uso domiciliar e controle de vetores de doenças. In: MARICONI, F. A.M. (Ed.) Insetos e outros invasores de residências. Piracicaba: FEALQ v. 6, 1999. p. 403-452.

FERRON, P. Biological control of insect pests by entomogenous fungi. Annual Review of Entomology, v. 23, n. 409-442, 1978.

FURLONG, M. J.; GRODEN, E. Evaluation of synergistic interactions between the colorado potato beetle (Coleoptera: Chrysomelidae) pathogen Beauveria bassiana and the insecticides, Imidacloprid, and Cyromazine. Journal of Economic Entomology, v. 94, n.2, p. 344-356, 2003.

GALLO,D.; NAKANO, O.; SILVEIRA NETO, S.; CARVALHO, R.P.L.; BAPTISTA, G. C.; BERTI, F. O. E.; PARRA, J. R. P.; ZUCCHI, R. A. A.; ALVES, S. B.; VENDRAMIN, J. D.; LOPES, J. R. S.; OMOTO, C. Entomologia Agrícola, Piracicaba: FEALQ 2002. 920 p. 10 v.

GARDNER W.; STOREY, G. K. Sensitivity of Beauveria bassiana to selected herbicides. Journal of Economic Entomology, v. 78, p. 1275-1279, 1985.

GEORGHIOU, G. P. Management of resistance in arthropods. In: Georghiou, G. P. \& T. Saito. Pest resistance to pesticides. New York: Plenum Pres, 1983. 509p.

The magnitude of the resistance problem. National Research Council. Pesticides resistance: strategies and tactics for management. Washington: National Academy Press, 1986. p. 14-43.

GRANDI, R. A. P., TRUFEM, S. F. B.; MARCELLI, M. P. Fungos. In: Biologia Vegetal. 5ed. (Raven, P. H., Evert, R. F.; Eichhorn, S. E. eds.). Rio de Janeiro: Guanabara Koogan. 1996. p. 192-225. (tradução).

HALL, H. M.; DUNN, P.H. The effect of certain insecticides and fungicides on pathogenic fungi to the spotted alfafa aphid. Journal of Economic Entomology, v. 52 p. 28-29, 1959.

IGNOFFO, C. M.; HOSTETTER, C.; GARCIA, C.; PINNELL, R. R. Sensivity of the entomopathogenic fungus Nomurea rileyi to chemical pesticides used on soybeans. Environmental Entomology, v. 4 p.765-768, 1975.

MALO, A.R. Estudio sobre la compatibilidad del hongo Beauveria bassiana (Bals.) Vuill.con formulaciones comerciales de fungicidas e insecticidas. Revista Colombiana de Entomología, Bogotá, v. 19, p. 151-158, 1993. 
MARQUINI, F.; PICANÇO, M. C.; GUEDES, N. C.; FERREIRA, S. F. Imidacloprid impact on arthropods associated with canopy of common beans. Neotropical Entomology, v. 32, n. 2, p. 335-342, 2003.

MIDIO, A. F.; SILVA, E. S. Inseticidas: acaricidas organofosforados e carbamatos. São Paulo: Roca, 1995.

MOINO JR., A.; ALVES, S. B. Efeito de Imidacloprid e Fipronil sobre Beauveria bassiana (Bals.) Vuill. e Metarbizium anisopliae (Metsch.) Sorok. e no comportamento de limpeza de Heterotermes tenuis (Hagen). Anais da Sociedade Entomológica do Brasil, Londrina, v. 27, n. 4, p. 611-619, 1998

MULOCK, B. S.; CHANDLER, L. D. Effect of Beauveria bassiana on the fecundity of western corn rootworm, Diabrotica virgifera virgifera (Coleoptera: Chrysomelidae). Biological Control, v. 22, p. 16-21, 2001.

NEVES, P. M. O. J.; ALVES, S. B. Controle associado de Cornitermes cumulans (Kollar, 1832) (Isoptera:Termitidae) com Metarbizium anisopliae, Beauveria bassiana e Imidacloprid. Scientia Agricola, Piracicaba, v. 56, n. 2, p. 305-311, 1999.

NEVES, P. M. O. J.; ALVES, S. B. Grooming capacity inhibition in Cornitermes cumulans (Kollar) inoculated with entomopathogenic fungi e treates with imidacloprid. Anais da Sociedade Entomológica do Brasil, Londrina, v. 29, n. 3, p. 537-545, 2000.

OLIVEIRA, C. N. D.; NEVES, P. M. O. J; KAWAZOE, L. S. Compatibility between the entomopathogenic fungus Beauveria bassiana and insecticides used in coffee plantations. Scientia Agricola, Piracicaba, v. 60, n. 4, p. 663-667, 2003.

OMOTO, C.; ALVES, S. B. Mecanismos de defesa de insetos contra entomopatógenos. In: ALVES, S. B. (Org.). Controle microbiano de insetos. 2 ed. Piracicaba: FEALQ p. $55-74,1998$.

OMOTO, C.Modo de ação de inseticidas e resistência de insetos a inseticidas. In: GUEDES, J. C.; COSTA, I. D., CASTIGLIONE, E. (Org.) Bases e técnicas do manejo de insetos. Santa Maria: Pallotti, p. 31-49, 2000.

ORIOLI, A. L; CAMARGO, A. J. A. Principais problemas ambientais causados pela implantação de lavouras. In: YOSHI, K; CAMARGO, A. J. A.; ORIOLI, A. L. (Org.) Monitoramento Ambiental nos projetos agrícolas do PROCEDER. Brasília: EMBRAPA Cerrados, JICA, 2000. p. 19-26.

PACHAMUTHU, P.; KAMBLE, S. T. In vitro study on combined toxicity of Metarbizium anisopliae (Hyphomycetes) strain ESC-1 with sublethal doses of cholorpyrofos, propetamphos and cyfluthrin against german cochroach (Dictyoptera: Blattellidae). Journal of Economic Entomology, v. 93 p. 60-70, 2000. 
PEDROSA-MACEDO, J. H. Manual de Pragas em Florestas - Pragas Florestais do sul do Brasil. IPEF/SIF, v. 2, 1993.112p.

PRIOR, C., JOLLANDS, P.; LE PATOUREL, G. Infectivity of oil and water formulations of Beauveria bassiana (Deuteromycotina: Hyphomycetes) to the cocoa weevil pest Pantorhytes plutus (Coleoptera: Curculionidae). Journal of Invertebrate Pathology, 52, p. 66-72, 1988.

QUINTELA, E. D. Effect of Imidacloprid on conidial germination and the pathogenicity of two entomopathogenic fungi to larvae of Diaprepes abbreviatus (Coleoptera: Curculionidae). 1996. 150 f. Ph.D. dissertation, University of Florida, Gainesville, FL, 1996.

QUINTELA, E. D.; MCCOY, C. W. Pathogenicity enhancement of Metharhizium anisopliae and Beauveria bassiana to first instars of Diaprepes abbreviatus (Coleoptera: Curculionidae) with sublethal doses of Imidacloprid. Environmental Entomology, v. 26, n. 5, p. 1173-1182, 1997.

QUINTELA, E. D.; MCCOY, C. W. Effects of Imidaclorid on development, mobility and survival of first instars of Diaprepes abbreviatus (Coleptera: Curculionidae). Journal of Economic Entomology, v. 90, p. 988-995, 1997.

QUINTELA, E. D.; MCCOY, C.W. Conidial Attachment of Metarhizium anisopliae and Beauveria bassiana to the larval cuticle of Diaprepes abbreviatus (Coleoptera: Curculionidae) Treated with Imidacloprid. Journal of Invertebrate Pathology, v. 72, n.3, p. 220-230, 1998a.

QUINTELA, E. D.; MCCOY, C. W. Synergistic effect of Imidacloprid and two entomopathogenic fungi on the behavior and survival of larvae of Diaprepes abbreviatus (Coleoptera: Curculionidae) in soil. Journal of Economic Entomology, v. 91, p. 110-122, 1998b.

REYNOLDS, S. E. The cuticule, growth regulators and moulting in insects: the essential background to the action of acylurea insecticides. Pesticide Science, v. 20, p. 131-146, 1987.

SHAH, P. A; PELL, J. K. Entomopathogenic fungi as biological control agents. Applied Microbiology and Biotechnology, v. 61, n.5-6, p. 413-423, 2003.

SOSA-GÓMEZ, D. R.; BOUCIAS, D. G.; NATION, J. L. Attachment of Metarbizium anisopliae to the southern green stink bug Nezara viridula cuticle and fungistatic effect of cuticular lipids and aldehydes. Journal of Invertebrate Pathology, v. 69, p. 31-39, 1997.

TAMAI, M. A.; ALVES, S. B.; LOPES, R. B.; FAION, M.; PADULLA, L. F. L. Toxicidade de produtos fitossanitários para Beauveria bassiana (Bals.)Vuill. Arquivos do Instituto Biológico, São Paulo, v. 69, n. 3, p. 89-96, 2002.

TOMIZAWA, M.; CASIDA, J. E. Selective Toxicity of Neonicotinoids Attributable to Specificity of Insect and Mammalian Nicotinic Receptors. Annual Review of Entomology, Berkeley, Estados Unidos. v.48, p. 339-364, 2003. 
VÁSQUEZ, C. A.; SALDARRIAGA, Y.; FABIO-PINEDA, G. Compatibilidade of the entomopathogenic fungus Beauveria bassiana with triflumuron. Revista Colombiana de Entomologia, Bogotá, v. 30, n. 1, p. 23-27, 2004.

WARE, G. W. An introduction to insecticides. Department of Entomology, University of Arizona, Tucson, Arizona. Disponível em: <http://ipmworld.umn.edu/chapters/ware.htm.>. Acesso em: 02 jan. 2010. 